\title{
Reflets
}

Revue ontaroise d'intervention sociale et communautaire

\section{La violence sexuelle contre les femmes dans les pays en guerre et vivant des conflits ethniques : défis pour la pratique}

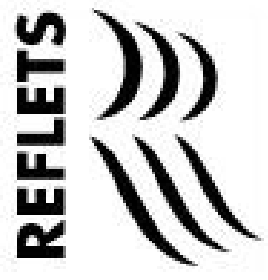

\section{Marie Lacroix et Charlotte Sabbah}

Volume 13, numéro 1, 2007

La violence dans tous ses états

URI : https://id.erudit.org/iderudit/016811ar

DOI : https://doi.org/10.7202/016811ar

Aller au sommaire du numéro

Éditeur(s)

Reflets : Revue ontaroise d'intervention sociale et communautaire

ISSN

1203-4576 (imprimé)

1712-8498 (numérique)

Découvrir la revue

Citer cet article

Lacroix, M. \& Sabbah, C. (2007). La violence sexuelle contre les femmes dans les pays en guerre et vivant des conflits ethniques : défis pour la pratique. Reflets, 13(1), 18-40. https://doi.org/10.7202/016811ar
Résumé de l'article

Des 27 millions de personnes réfugiées et déplacées dans le monde aujourd'hui, 80 \% sont des femmes et des enfants. Entre les années 1992 et 2001, 116709 femmes ont demandé l'asile au Canada. La violence sexuelle contre les femmes dans un contexte de guerre et de conflits ethniques est considérée comme une violation des droits humains depuis les années 90. Cette problématique interpelle la communauté internationale et toutes les praticiennes. À leur arrivée, les femmes réfugiées victimes de violence sexuelle n'ont pas accès à des services sociaux adéquats, adaptés à leurs besoins. Cet article explore les dimensions du viol de guerre et de la victimisation de femmes qui peuvent éprouver des problèmes d'intégration lors de leur installation au pays à cause de leur grande vulnérabilité et des séquelles des violences subies. De plus, notre conception occidentale des secteurs "privé » et " public » soulève d'importantes questions sur nos approches d'intervention qui ne sont pas nécessairement appropriées aux besoins de ces femmes.
Tous droits réservés ( $)$ Reflets : Revue ontaroise d'intervention sociale et communautaire, 2007
Ce document est protégé par la loi sur le droit d'auteur. L'utilisation des services d’Érudit (y compris la reproduction) est assujettie à sa politique d'utilisation que vous pouvez consulter en ligne. 


\section{La violence sexuelle contre les femmes dans les pays en guerre et vivant des conflits ethniques : défis pour la pratique}

Marie Lacroix, PhD.

Professeure adjointe, École de service social, Université de Montréal

et

\section{Charlotte Sabbah}

Professeure en service social, CEGEP St-Jean-sur-Richelieu et chargée de cours, École de service social, Université de Montréal

\section{Introduction}

"Le viol de guerre ou lors de conflit ethnique est la plus répandue des formes de violence contre les femmes, quoique paradoxalement très peu conпue et rapportée. "
Le viol de guerre ou lors de conflit ethnique est la plus répandue des formes de violence contre les femmes, quoique paradoxalement très peu connue et rapportée. C'est un des types de violence qui motive les femmes à fuir leur communauté, augmentant du coup les groupes de personnes déplacées et réfugiées, soit à l'intérieur même du pays d'origine, soit dans un pays d'accueil comme le Canada. En tant qu'Occidentaux, nous nous devons de nous pencher sur le sort de ces femmes établies au Canada.

Les événements internationaux nous interpellent en tant que société d'accueil canadienne. La condition des femmes réfugiées ayant subi de la violence sexuelle dans un contexte de guerre ou de conflits ethniques sera ici utilisée pour démontrer comment les réalités internationales exigent que nous analysions de manière critique le fondement de nos connaissances, nos méthodes d'interventions et notre pratique proprement dite. 
"C'est depuis le début des années 90, et comme suite à la publication de multiples rapports internationaux, qu'a émergé l'impératif de documentation du problème $d u$ viol des femmes dans un contexte de guerre et de conflits ethniques, avec celui de sa compréhension comme une question de droits humains. »
Nous nous intéresserons tout d'abord aux problématiques prédominantes reliées aux expériences du viol de guerre sur les femmes, à leurs séquelles, à leurs impacts dans le temps et à la capacité de ré établissement ${ }^{2}$ de ces femmes dans un contexte canadien, malgré leur grande vulnérabilité. L'articulation du "privé " et du "public " soulève de nouvelles questions sur nos approches occidentales et exige donc une quête de nouvelles formes d'intervention. Cet article est basé sur une importante recension des écrits sur le sujet et sur des informations recueillies auprès de dix informateurs-clés travaillant avec des femmes réfugiées à Montréal. Ces entrevues font partie d'une recherche préliminaire sur les conséquences de la violence basée sur le genre envers les femmes réfugiées dans un contexte de guerre ou de conflits ethniques.

Nous sommes en mesure de dégager de notre recension des écrits trois thèmes reliés et autour desquels pivote la compréhension de la violence sexuelle dans un contexte de guerre et de conflits ethniques: 1) le contexte moderne des guerres et des conflits ethniques; 2) la définition de la violence contre les femmes selon un cadre intersectionnel de relations de genre et de constructions culturelles; 3) les conséquences psychologiques, sociales et physiques de la violence sexuelle et les implications pour la pratique. Ces thèmes fondamentaux seront à la base du développement de nouvelles approches et pratiques et constitueront le cadre théorique des futures recherches.

\section{Le contexte international de l'asile}

C'est depuis le début des années 90, et comme suite à la publication de multiples rapports internationaux, qu'a émergé l'impératif de documentation du problème du viol des femmes dans un contexte de guerre et de conflits ethniques, avec celui de sa compréhension comme une question de droits humains. Alors que ce type de violence a été largement pratiqué au cours de l'histoire (Hynes et Lopes Cardoso 2000) et plus récemment documenté comme pratique répandue au Mozambique, au Rwanda, en Bosnie, au Kosovo, au Pérou et au Liberia (Kirk 1992), ce n'est qu'avec la guerre en ex-Yougoslavie et lors du génocide du Rwanda que 
"La reconnaissance de cette situation en tant que motif valable pour l'obtention du statut de réfugié demeure une préoccupation majeure pour les organismes internationaux de défense des droits humains et de défense des droits des fermmes. Cette absence de reconnaissance influence non seulement le traitement de la détermination $d u$ statut, mais également les mesures d'accueil dont ces femmes pourraient bénéficier dans des circonstances différentes (Schafer 2002; Newland 2004). " l'on reconnait ce type de violence comme arme idéologique et politique de guerre. Cette problématique avait été jusque-là tue et minimisée comme élément indissociable et inévitable de la guerre. À la suite de la guerre en Bosnie, le travail du Tribunal criminel international contribua à l'inclusion du phénomène dans un agenda sur les droits humains. Son travail révéla alors une "absence substantielle de connaissances sur ce phénomène " (Skjelsbaek 2001 : 212; Buss 2002). Le Tribunal décréta que «le viol des femmes musulmanes yougoslaves était un crime contre l'humanité " (Buss 2002). La violence sexuelle a déjà été décrite comme un crime obscène (Skjelsbaek 2001); le viol de guerre comme une violence extrême (Niarchos 1995); en termes juridiques, cette violence est définie comme un acte politiquement motivé, une violation des droits humains (Mahmud 1996; Arcel 1998) et un crime contre l'humanité (Buss 2002; Masson 1999) et devrait être traité en tant que tel (Biehler 2002).

Cependant, en dépit de la documentation importante fournie sur les raisons motivant la fuite des femmes violées ou torturées (Nduwimana 1999;Valji \& De La Hunt 1999; Atlani \& Rousseau 2000; Batinic 2001; Schafer 2002) et aussi du fait que nous sachions maintenant que le viol et la violence sexuelle sont des instruments politiques et idéologiques de guerre (Salzman 1998; Gardam \& Charlestown 2000; Hynes et Lopez Cardoso 2000; Hynes 2004), la question du droit d'asile des femmes victimes de violence organisée soulève encore de nombreuses questions sur le plan international.

La reconnaissance de cette situation en tant que motif valable pour l'obtention du statut de réfugié demeure une préoccupation majeure pour les organismes internationaux de défense des droits humains et de défense des droits des femmes. Cette absence de reconnaissance influence non seulement le traitement de la détermination du statut, mais également les mesures d'accueil dont ces femmes pourraient bénéficier dans des circonstances différentes (Schafer 2002; Newland 2004). 


\section{Le contexte contemporain des guerres et des conflits}

Tel qu'expliqué par Sidéris, le "tissu social des pays en guerre est déchiré lorsque la population civile et ses structures sociales et culturelles sont visées. Les guerres modernes détruisent de ce fait, l'organisation et les relations sociales sécurisantes, stabilisantes, qui sont source de dignité humaine » (2002 : 715). La compréhension de la détresse subjective du viol de guerre doit donc passer par celle du déchirement du contexte social de son tissu social ainsi que de la compréhension de l'interdépendance des processus psychologiques et des environnements sociaux. À cet effet, Sideris (2003) observe dans son travail sur les conflits au Rwanda, que le viol est ancré dans l'extrémisme hutu comme un instrument de vengeance ethnique.Alors qu'il est simultanément un instrument de torture politique stratégique visant la démoralisation et l'humiliation de l'ennemi, il reflète et engendre également dans l'identité masculine hutu une série de rapports complexes entre les sexes, et cela, indépendamment du statut économique des hommes (Baines 2003 : 488).

\section{L'envergure du problème}

Le Haut Commissariat pour les réfugiés des Nations Unies (HCR) a rapporté que la violence sexuelle systématique envers les femmes et les fillettes constitue l'une des causes les plus importantes de la fuite des femmes réfugiées. (www.unhcr.org, 8 mars 2004). Selon Jacobson (1999: 182), « le personnel du HCR devrait présumer la violence basée sur le genre jusqu'à preuve du contraire ». Les praticiennes que nous avons interviewées confirment le bien-fondé de cette orientation dans leur travail avec les femmes provenant de pays où il est documenté que se pratique ce type de violence.

\section{Le cadre $d$ 'analyse intersectionnelle des relations de genre et de constructions culturelles}

Selon Jacobson (1999), quoique le problème du viol des femmes soit d'une grande envergure, notre travail d'intervention et d'analyse ne doit pas être limité aux femmes violentées seulement. Les 
"Les chercheuses

féministes insistent

sur l'importance

d'une perspective de genre pour l'analyse des configurations historiques des relations sociales entre les genres dans les pays d'origine."

\section{"Farwell ajoute que} "la violence sexuelle de guerre se bâtit sur des relations de genre préexistantes, et donc sur des dynamiques socioculturelles. Ce qui rend le viol un instrument de guerre si terrifiant et si efficace est qu'il s'attaque directement au concept d'honneur, situé dans le corps des femmes " (2004 : 394).» chercheuses féministes insistent sur l'importance d'une perspective de genre pour l'analyse des configurations historiques des relations sociales entre les genres dans les pays d'origine. Les relations entre les sexes définissent en fait la place familiale des femmes, leurs statuts vis-à-vis leurs enfants, les hommes et les aînés, ainsi que leur rôle économique et obligations religieuses (Sideris 2003).Tel qu'il a été mentionné par Baines (2003), notre compréhension du phénomène doit se baser sur les raisons évoquées par les membres de ces sociétés et les solutions au problème doivent provenir de leurs narrations sociales et politiques. Par exemple, une étude sur la violence sexuelle en Ouganda démontre que "les soldats ougandais considèrent la femme comme une propriété. [...] C'est ainsi qu'en aliénant le travail politique des femmes, ils espèrent priver l'ennemi du fruit de leur labeur" (Turshen 2000 : 813).

Le viol comme une arme de guerre reflète le manque de respect des droits reproductifs humains. (Rojnik, AndoleskJeras, Obersnel-Kveder 1995 : 312; Arcel 1998; Baines 2003). Il confirme pour Mahmud (1996) le statut subordonné des femmes dans la vie publique et privée, donc leur statut social de seconde classe. Farwell ajoute que "la violence sexuelle de guerre se bâtit sur des relations de genre préexistantes, et donc sur des dynamiques socioculturelles. Ce qui rend le viol un instrument de guerre si terrifiant et si efficace est qu'il s'attaque directement au concept d'honneur, situé dans le corps des femmes " (2004:394). Les militaires ne font qu'opérationnaliser des systèmes qui font violence à la femme : « la complexité des inégalités entre les sexes, de classes, les inégalités raciales et d'ethnicité, l'impérialisme, la colonisation et le néo-colonialisme sont à la base de la violence militaire contre les femmes " (Okazawa-Rey 2002 : 374). À cette liste, nous pouvons ajouter le concept de nation, l'économie véhiculée par la mondialisation et les autres types de violences étatiques. Selon Farwell (2004), le continuum de la violence contre les femmes est alimenté par l'intersection de toutes ces dimensions dans les contextes patriarcaux. C'est "par la transgression du viol et de la torture que le corps prend toute sa signification en relation avec le pouvoir et le désir et qu'il est sexualisé, différencié et racialisé " (Baines 2003 : 489). Sa fonction ultime est "le 
"Le travail social avec ces femmes doit donc tenir compte du fait que le viol de guerre est au Canada un motif valable d'asile, d'autant plus que $48 \%$ des personnes réfugiées assistées par le gouvernement et réinstallées ou revendicatrices d'asile au pays sont des femmes qui peuvent avoir été violées." maintien du contrôle social par la régularisation de la sexualité, et par la privation des droits fondamentaux humains " (Baines 2003 : 483).

Le Canada a été le premier pays à mettre en vigueur de nouvelles directives sur la persécution basée sur le genre en 1993 à la Commission de l'immigration et du refuge (CISR). Quoique plusieurs femmes arrivent souvent en tant que revendicatrices du statut de réfugié, elles arrivent également en tant que réfugiées parrainées ou assistées par le gouvernement. Le travail social avec ces femmes doit donc tenir compte du fait que le viol de guerre est au Canada un motif valable d'asile, d'autant plus que $48 \%$ des personnes réfugiées assistées par le gouvernement et réinstallées ou revendicatrices d'asile au pays sont des femmes qui peuvent avoir été violées.

\section{Des défis pour la pratique : Conséquences physiques, psychologiques et sociales de la violence sexuelle dans un contexte de guerre et de conflits ethniques}

Nous avons réalisé dix entrevues avec des intervenantes travaillant auprès de femmes réfugiées dans des organismes communautaires ou, pour l'une d'entre elles, dans un hôpital. Les questions du guide d'entrevue ont été élaborées à partir des thèmes recensés dans notre revue de littérature et qui nous apparaissaient non seulement importants pour une compréhension globale du phénomène, mais aussi pertinents pour le développement de pratiques répondant aux besoins de ces femmes. Ainsi, les catégories d'analyse sont celles qui non seulement sont soulevées dans la littérature, mais qui se dégagent aussi de la narration des intervenantes que nous avons rencontrées.

\section{Les conséquences physiques}

Les blessures physiques en tant que conséquences de la violence sexuelle prévalent dans les rapports internationaux. S'y rajoutent les maladies transmises sexuellement et leVIH/Sida dont peuvent souffrir les immigrantes illégales et les réfugiées. Par exemple, 
"Par exemple, $80 \%$ des femmes rwandaises violées pendant le génocide ont été diagnostiquées séropositives ou atteintes du Sida (Nduwimana, décembre 2004).»
$80 \%$ des femmes rwandaises violées pendant le génocide ont été diagnostiquées séropositives ou atteintes du Sida (Nduwimana, décembre 2004). En Sierra Leone, les Physicians for Human Rights (PHR 2000) se plaignent d'un manque d'infrastructures qui les empêche d'aider les femmes souffrantes de traumatismes sévères et reliés au viol. Il est clair que ces femmes peuvent arriver au Canada avec de graves et complexes problèmes de santé. D'après Duchesneau (2004: 48), " les immigrants illégaux et les réfugiés atteints du VIH/SIDA ont des problèmes complexes qui exigent une compréhension de base des droits humains, de l'immigration, de la politique internationale, des traumatismes des réfugiés et de leur résilience et de la maladie du VIH ». Lorsque les femmes revendicatrices du statut de réfugié arrivent enceintes au pays d'accueil, les praticiens devraient se préoccuper de l'origine de cette grossesse au cas où elle aurait été non désirée et que le nourrisson soit rejeté à sa naissance (Carpenter 2000; Hynes et Lopez Cardoso 2000; PHR 2000; Swiss et al. 1998). Comme le souligne une intervenante, il faut savoir décoder les signes :

"Le premier indice est le pays d'origine de la femme, s'il s'agit d'un pays en guerre comme le Rwanda ou le Burundi avant ou la République démocratique du Congo et que la femme fait de l'insomnie, montre des signes de déprime ou de syndromes post-traumatiques. »

Nous ne connaissons pas l'envergure de situations de HIVSIDA chez les réfugiées et revendicatrices du statut au Canada. En 2006 cependant, à la Conférence internationale sur le SIDA tenue à Toronto, une équipe de chercheurs (Wong et al.2006) a remarqué l'interrelation des déterminants biophysiques, psychologiques, socioéconomiques et sociopolitiques dans l'état mental des immigrants et des réfugiés. En plus de gérer leur maladie du HIVSIDA, les personnes interviewées par les chercheurs se trouvaient confrontées à des situations de racisme, de logement inadéquat, de pauvreté et d'isolement social. Les répondantes de notre étude s'entendent sur le fait que certaines femmes « cachent bien leur détresse et qu'il est difficile de deviner à tous les coups. Certaines 
qui apprennent leur séropositivité lors des examens médicaux pour l'immigration sont encore plus dévastées » (intervenante).

Les répondantes de notre étude relèvent certains signes d'alarme facilitant l'évaluation des situations de violence sexuelle. Ces indices se retrouvent aussi dans les travaux internationaux dans ce domaine.

"Quand elles se plaignent souvent de maux de ventre, font des cauchemars, bégaient, montrent une tristesse, une colère ou une agressivité extrême, un manque de contrôle, c'est un signe. D'autres cachent leur détresse derrière un masque de bonheur et d'exubérance, en rigolant beaucoup pour ne pas montrer leur peine. Les démarches d'immigration les obligent à faire des examens médicaux et avec des diagnostics de maladies transmises sexuellement ou de VIH, on les réfere alors à la clinique $X$ pour un suivi plus approprié."

"Lorsque ces immigrantes et réfugiées avaient demandé de l'aide, plusieurs avaient été retraumatisées alors qu'elles cherchaient des services spécifiques de leurs besoins dans un système où les services sociaux et de santé n'étaient pas coordonnés. Cette situation les forçait à répéter plusieurs fois leur pénible histoire aux professionnelles des differents services. "
Dans l'étude de Wong et al. (2006), les femmes immigrantes et réfugiées ont exprimé leur crainte de la maladie en l'absence de soutien pour s'occuper de leurs enfants. Lorsque ces immigrantes et réfugiées avaient demandé de l'aide, plusieurs avaient été retraumatisées alors qu'elles cherchaient des services spécifiques de leurs besoins dans un système où les services sociaux et de santé n'étaient pas coordonnés. Cette situation les forçait à répéter plusieurs fois leur pénible histoire aux professionnelles des différents services.

\section{Les conséquences psychologiques}

La guerre et les conflits ethniques engendrent souvent le déplacement à l'intérieur du pays, la fuite et l'expérience des camps de réfugiés ou la fuite et le ré établissement dans un pays tiers. Les recherches d'Eisenbruch, Jong \& Van de Put (2004) réaffirment la réalité que les réfugiés vivent en général. Ils soulignent une série de situations traumatisantes même lorsque les réfugiés se réfugient dans des camps, lors d'un ré établissement, ou d'un 
«..."les réfugiés, même après leur ré établissement, vivent des deuils culturels et personnels qui seront exprimés plus tard. Ces observations ont des implications importantes non seulement sur les théories du trauma, mais également sur les pratiques d'intervention en santé mentale »..." rapatriement et réinstallation dans le pays d'origine. Quoique cette dernière leur permet de fuir les horribles réalités de vie des camps, " elle n'apporte pas nécessairement la paix et le soulagement " (Eisenbruch et al. 2004 : 128).

Les déplacements successifs sur la route de l'asile à la recherche de sécurité et de paix ont des impacts divers sur l'expérience traumatique des femmes ayant subi des formes de violence organisée et des viols de guerre. Certaines sont de nouveau violées dans des camps de réfugiés, par les militaires ou les gardiens, ou peuvent être victimes des passeurs dont le rôle est pourtant de les aider à fuir. Au viol peuvent s'ajouter différentes sortes de mauvais traitements ou formes de chantage. Nous observons qu'elles hésitent à leur arrivée au Québec à dénoncer leurs agresseurs par peur de représailles. Pour Brittain (2002 : 45), les conflits armés entraînent de nouvelles formes d'exploitation des femmes qui, pour fuir l'horreur et sauver leur peau, se retrouvent plongées dans de nouveaux pièges avec de nouveaux agresseurs. LoiselleLéonard (2002:29) explique que ces exactions sont d'autant plus bouleversantes qu'elles sont commises par des personnes de confiance ou en autorité. Cette détresse peut perdurer longtemps après l'arrivée dans le pays d'accueil. Ces femmes violentées n'ont pas la chance, comme d'autres femmes plus fortunées, de vivre leur fuite comme une délivrance malgré les dangers du périple migratoire.

Eisenbruch et al., qui ont conduit ses recherches dans les camps de réfugiés, insistent sur le fait que "les réfugiés, même après leur ré établissement, vivent des deuils culturels et personnels qui seront exprimés plus tard. Ces observations ont des implications importantes non seulement sur les théories du trauma, mais également sur les pratiques d'intervention en santé mentale" (2004:128). C'est ce que les intervenantes que nous avons interviewées ont également observé. Les réfugiées ou revendicatrices du statut n'expriment pas immédiatement la détresse reliée à leurs traumatismes. Elles sont en proie au stress dû aux procédures problématiques de l'immigration et de la citoyenneté et sollicitées par leur survie et activités quotidiennes, 
telles que leur insertion sur le marché du travail,leurs responsabilités et obligations maternelles et matérielles :

"Les femmes qui arrivent au Canada pour revendiquer le statut de réfugié doivent se plonger tout de suite après leur arrivée dans le processus et les démarches. C'est une grosse machine et elles n'ont pas le choix de plonger dedans si elles veulent être acceptées. Donc, qu'elles le veuillent ou pas, prêtes ou non, elles doivent embarquer et mettre leur détresse de côté. Certaines aussi sont plus préoccupées par leurs papiers, la réunification familiale et le sort de la famille laissée derrière. Elles s'intègrent, car elles n'ont pas le choix, si elles veulent rester ici et c'est très difficile."

\section{Le dévoilement du viol et ses conséquences}

Il est important de noter que le silence des sentiments de honte et de culpabilité a été imposé (Amnesty International 2004;

"Lorsque les

femmes décident de parler, les écrits rapportent qu'elles sont personnellement rejetées par leur famille qui peut également rejeter leurs enfants nés du viol, ou qu'elles sont stigmatisées par leur communauté (Chester 1992). " Jacobson 1999; Mackay 2000; Skjelsbaek 2001. Lorsque les femmes décident de parler, les écrits rapportent qu'elles sont personnellement rejetées par leur famille qui peut également rejeter leurs enfants nés du viol, ou qu'elles sont stigmatisées par leur communauté (Chester 1992). Les praticiennes doivent donc être attentives aux conditions de dévoilement qui peuvent être freinées par ces dangers :

Dans toutes les sociétés, y compris les nôtres dites occidentales, chaque fois qu'il est question de viol, beancoup ont tendance à rendre la femme coupable, responsable. Dans les pays d'origine de ces femmes, c'est encore pire. La femme est mise au banc des accusées, elle est maudite, c'est la honte pour la famille et la communauté. Les femmes ont alors peur d'être rejetées, stigmatisées. Elles se sentent coupables et ont peur de parler. Parfois aussi, elles ont tellement mal qu'il leur est impossible de sortir les mots de leur bouche. 
"L'obstacle le plus important est le lien de confiance. La plupart des femmes nous associent aux autorités de l'immigration et ont peur, à tort, d'une délation de notre part. C'est très difficile et frustrant pour nous, les intervenantes, parce que notre premier souci, c'est de les aider et de les protéger, pas de leur créer des problèmes; mais, elles ont du mal à croire et à comprendre cela, je ne sais pas pourquoi."
Ainsi, la manière de survivre à la violence individuelle ou collective de toute une communauté est conditionnée par les valeurs culturelles, sociales et économiques ainsi que par les rapports de genre. Compte tenu de ce cadre de référence, la violence entraîne la perte d'appartenance et d'identité sociales et la perte de la culture dans un sens large (Sideris 2003 : 716). Lorsque toute une communauté a été touchée par les conflits ethniques, comme pendant le génocide du Rwanda, les traumatismes et les symptômes subséquents peuvent avoir un impact sur toute la communauté.

\section{Les obstacles au dévoilement et les stratégies utilisées par les intervenantes}

Les répondantes de notre recherche observent les difficultés reliées au dévoilement du viol. Les femmes violées éprouvent du dégoût pour elles-mêmes et de la culpabilité parce qu'elles se sentent responsables de ce qu'il leur est arrivé à cause de comportements qu'elles qualifient d'inadéquats en présence de leurs bourreaux et agresseurs. Selon Loiselle-Léonard (2002 : 29), le sentiment de honte et de culpabilité, tout comme la stigmatisation que le viol engendre chez la femme et sa famille, incite de façon tacite ou explicite au suicide, à l'exode ou à la disparition pour épargner la communauté. Il est donc impératif et primordial pour les intervenantes d'établir avec ces femmes un lien de confiance solide qui facilitera la mise en perspective de la violence et du dévoilement du viol.

"L'obstacle le plus important est le lien de confiance. La plupart des femmes nous associent aux autorités de l'immigration et ont peur, à tort, d'une délation de notre part. C'est très difficile et frustrant pour nous, les intervenantes, parce que notre premier souci, c'est de les aider et de les protéger, pas de leur créer des problèmes; mais, elles ont du mal à croire et à comprendre cela, je ne sais pas pourquoi." 
"Cela peut prendre des mois, dans certains cas des années, mais les barrières ne sont jamais franchies. Je respecte la zone de confort des femmes; si elles décident d'en parler, la porte de mon bureau leur est toujours ouverte, mais je ne les force pas, par principe."

Cela signifie que les symptômes post-traumatiques peuvent persister et perdurer pendant un certain temps et que les intervenantes doivent patiemment attendre que les femmes soient prêtes à en parler.

Pour contourner les nombreux obstacles qu'elles rencontrent à l'intervention, certaines intervenantes choisissent d'ouvrir le dialogue grâce à des moyens détournés comme les conversations anodines, et même des jeux de rôles, afin de mettre leurs clientes en confiance. Cependant, selon nos répondantes, il faut que celles qui travaillent en première ligne soient conscientes de la centralité du viol dans la demande de statut de ces femmes et qu'elles s'assurent de la préparation adéquate de l'avocat qui les représente. Selon une praticienne montréalaise, son expérience lui a appris qu'elle peut présumer que les revendicatrices d'asile qui ont été détenues ou emprisonnées ont également été victimes de multiples viols. Elles se sentent souvent honteuses ou coupables, surtout si elles sont devenues enceintes et qu'elles doivent retourner chez elles avec l'enfant (Bassolé et al. 2004). Les mêmes observations ont été documentées chez les femmes réfugiées du Mozambique et du Rwanda (Sideris 2003).

Il est fréquent que ces femmes ne rencontrent les services communautaires spécialisés que lors du processus de détermination, qu'elles se sentent en confiance et qu'elles révèlent enfin leur viol, ce qui exige alors des praticiennes une grande acuité et sensibilité dans l'exploration du problème. Pour faciliter le dévoilement, certaines normalisent le viol en tenant compte du contexte social duquel les femmes proviennent lorsque la pratique du viol y est généralisée, ou elles utilisent le moment de remplir le formulaire d'immigration, une stratégie neutre et indirecte, qui permet aux femmes qui sont psychologiquement prêtes à le faire, de dévoiler le ou les viols subis. 
"Le processus de détermination, sa lenteur et son incertitude inhérente aggravent l'expérience du déracinement (Lacroix 2004)."
"Le remplissage des formulaires d'immigration est aussi un moyen efficace, car les fermmes, en dévoilant certaines informations, arrivent à se confier. De plus, c'est un moyen neutre qui ne suppose pas une conversation directe. Mais certaines femmes insistent pour remplir seules leurs formulaires et on respecte leur choix. L'accompagnement à l'audience est aussi un autre moyen, car les femmes dévoilent la majeure partie de leur histoire devant le commissaire à l'Immigration."

"Les horreurs vécues par leurs proches (parents, amis, collègues, voisins, professeurs) en l'absence de soutien familial, dans une culture où la communauté et la solidarité sont primordiales, rendent les femmes violentées méfiantes de tout type d'autorité gouvernementale et du personnel qui la représente (Bassolé et al.2004). Pour Loiselle-Léonard (2002 : 23), cette souffrance sociale est "engendrée par des rapports humains opprimants, abusifs et violents, à des relations qui font mal, tourmentent et endommagent moralement et/ou physiquement les individus et les groupes sociaux qui les subissent ".

Dans une étude récente sur la traite des personnes au Canada (Oxman-Martinez, Lacroix \& Hanley 2005), plusieurs praticiennes, surtout montréalaises, ont admis ne pas être au courant de la prévalence de la traite des femmes et des enfants dans leur milieu de travail. Prisonnières de leurs positions idéologiques quant à la définition de la traite, ces professionnelles ne posaient simplement pas la question qui aurait permis aux femmes victimes un traitement adéquat.

Le processus de détermination, sa lenteur et son incertitude inhérente aggravent l'expérience du déracinement (Lacroix 2004). Les deuils et le sentiment de dislocation ajoutent une autre dimension et un autre fardeau que les femmes doivent porter (Bassolé et al.2004). Étant donné que ces femmes revendicatrices 
du statut de réfugié veulent d'abord et avant tout régler le problème de leur statut pour se sentir en sécurité et se stabiliser dans un environnement protecteur, il n'est peut-être pas toujours approprié d'insister sur une révélation qui risquerait de mettre à l'épreuve leur déséquilibre psychologique, alors qu'elles sont déjà vulnérables. C'est ainsi que certaines praticiennes décident de ne s'enquérir des possibilités du viol que lorsque la demande de statut a été rejetée et qu'il n'existe aucune autre alternative pour éviter la déportation. Plusieurs pensent d'ailleurs que les femmes découvrent avec le temps des mécanismes de survie et se concentrent sur l'avenir pour elles-mêmes et leurs enfants (voir Bassolé et al. 2004).

Bien que la plupart des études identifient la stigmatisation et la honte comme les obstacles les plus importants au dévoilement, Weaver (2005) nous rappelle qu'elle a appris lors de son étude auprès de femmes réfugiées tamoules qu'il faut toujours réexaminer ce que nous pensons connaitre et nos manières de nous comporter. En présence des membres de leur famille, dans un cadre qui leur permettait de remettre en question nos notions occidentales de l'intimité et de la confidentialité, ces femmes se sentirent soulagées lorsqu'elles révélèrent les violences sexuelles et les autres formes de violence dont elles ont été victimes.

\section{Le cadre clinique}

Selon nos répondantes, le cadre institutionnel ne leur permet pas de fonctionner de manière satisfaisante.

"Elles sont débordées de travail et n'ont pas toujours la disponibilité nécessaire pour accorder aux femmes toute l'attention dont elles auraient besoin pour parler de leurs expériences, de vider leur sac. Parfois aussi, c'est difficile de trouver le moment opportun, l'intimité qu'il faut, car il y a beaucoup d'autres personnes dans la maison. "

Une seule des intervenantes interviewées se sentait adéquatement outillée pour intervenir auprès de la clientèle 
"Les intervenantes ne se sentent

généralement

pas assez bien

préparées ou formées

pour accompagner les

survivantes de violence organisée en situation de conflits armés." spécifique que constituent les femmes réfugiées qui ont connu l'expérience traumatique de la violence organisée et du viol en contexte de conflits armés. Elle attribuait cela à sa participation au travail d'une équipe multidisciplinaire très au fait des questions interculturelles et qui organisait régulièrement des rencontres et des séminaires de formation et du coaching pour les intervenantes qui en manifestaient le besoin. Les autres intervenantes, même si elles étaient unanimes pour reconnaitre ne pas disposer d'un cadre clinique formel leur permettant de dépister le viol de guerre chez leurs clientes, pensaient pouvoir se fier à leur expérience et à leur intuition pour présumer, sans trop de marge d'erreur, les traumatismes chez certaines femmes.

Les intervenantes ne se sentent généralement pas assez bien préparées ou formées pour accompagner les survivantes de violence organisée en situation de conflits armés. Elles se sentent démunies et mal outillées pour l'intervention auprès de victimes à cause de leur méconnaissance des contextes politiques, sociaux, économiques et culturels dans lesquels surviennent les viols. De plus, la relation de confiance nécessaire à l'établissement d'une alliance thérapeutique avec leurs clientes exige du temps et de la patience que leur charge de travail trop importante ne leur permet pas de fournir.

"Nous recevons aussi beaucoup de soutien et de formation de la part de professionnels qualifiés ayant l'expertise avec ce type de clientèle, dans un groupe restreint et fermé formé d'intervenants travaillant dans le milieu. Nous recevons de la supervision aussi. Mais, malgré toute cette expérience et ces formations, il arrive toujours des situations qui viennent nous chercher. On se sent totalement perdues et désemparées, on ne sait pas quoi faire, comment aider, comment se comporter devant tant de détresse. On ne sait pas quels mots utiliser pour consoler ou soutenir. On se dit intérieurement qu'on va réussir, mais on rate notre coup." 
"Il leur est très difficile d'évaluer les interventions et leurs impacts sur leurs clientes. Pour certaines intervenantes, les succès

et progrès des femmes sont dus à leur grande capacité de résilience. D'autres estiment que toutes les femmes ne maintiennent pas le contact avec elles par choix et surtout par désir de tourner la page, ce qui rend impossible toute forme d'évaluation."
Il leur est très difficile d'évaluer les interventions et leurs impacts sur leurs clientes. Pour certaines intervenantes, les succès et progrès des femmes sont dus à leur grande capacité de résilience. D'autres estiment que toutes les femmes ne maintiennent pas le contact avec elles par choix et surtout par désir de tourner la page, ce qui rend impossible toute forme d'évaluation. Enfin, les interventions sont continues et multidisciplinaires incluant travailleuses sociales, médecins, psychiatres et psychologues.

Bien qu'elles s'informent parfois de leur propre chef, par conscience professionnelle et dévouement à leur profession, toutes les praticiennes répondantes ont cependant insisté sur la nécessité d'une meilleure connaissance du contexte culturel, social et politique des pays d'origine des réfugiées ayant connu des violences et viols de guerre.

\section{Les approches occidentales et les obstacles institutionnels}

Eisenbruch note que "les événements traumatisants sont transcendés par des expériences historiques et médiatisés par une culture locale $[\ldots]$ et que la compréhension des expressions de la détresse d'une communauté facilite le décodage clinique du profil symptomatique des désordres psychologiques et sociaux " (Eisenbruch et al.2004 : 128). Il est donc suggéré de tenir compte de l'intégration émotionnelle et personnelle réalisée par les femmes victimes dans la détermination des services spécialisés. Il est important de mentionner ici le manque d'unanimité actuelle dans le domaine de la santé mentale sur les critères d'évaluation occidentaux du syndrome post-traumatique: «le débat se poursuit sur la permanence des symptômes post-traumatiques des survivants de violence communautaire [...] et sur l'acuité des critères d'évaluation du SPT de la torture et des traumatismes " (Eisenbruch, Jong and Van de Put 2004 : 123).

Il faudrait donc plutôt concentrer nos efforts sur la compréhension du contexte local duquel les réfugiées et revendicatrices proviennent et selon une vision intersectionnelle. Selon Sideris, "la destruction de l'ordre social et culturel se manifeste dans les formes subjectives de la détresse qui à leur tour reflètent 
"Les recherches menées dans les camps de réfugiés ont souligné la nécessité de réviser la compréhension occidentale du viol dans un contexte de guerre et de violence organisée et de réévaluer la validité de certains modèles d'intervention pratiqués par les praticiens occidentaux..." l'interdépendance des processus psychologiques et des environnements sociaux, facteurs largement négligés par le discours biomédical » (2003 : 717). Cette situation nous amène inévitablement à une critique du discours biomédical dominant. Elle nous oblige également à critiquer les instruments d'évaluation occidentaux tels que les indicateurs de stress post-traumatique et les modes de traitement comme les thérapies verbales et la médication. La compréhension de l'influence des interventions de type occidental signifie également « que les professionnelles de la santé se doivent une meilleure maitrise des raisons culturelles qui motiveraient une femme victime de violence de surmonter sa souffrance ou pas " (Neill 2000).

Les femmes réfugiées qui arrivent au Canada après avoir vécu l'expérience de la violence sexuelle sont souvent confrontées à des professionnelles de la santé et des services sociaux n'ayant pas encore d'expertise dans ce domaine. Les recherches menées dans les camps de réfugiés ont souligné la nécessité de réviser la compréhension occidentale du viol dans un contexte de guerre et de violence organisée et de réévaluer la validité de certains modèles d'intervention pratiqués par les praticiens occidentaux (Atlanti \& Rousseau 2000). Jennings et Swiss (2000) pensent que les concepts doivent être définis, nommément par la participation sémantique des participants, afin d'éviter les biais culturels et de maximiser l'intégration des cultures locales dans le développement d'instruments.

Par ailleurs, quant à l'accessibilité des ressources, il est reconnu par nos répondantes que la complexité et la difficulté de l'intervention auprès des femmes victimes de viol et de violence organisée en contexte de conflits armés pose la problématique de l'adaptabilité des services offerts aussi bien dans le réseau institutionnel que dans le milieu communautaire. Cette problématique se manifeste sur le plan des structures publiques et des organisations communautaires, ainsi que sur celui de contraintes budgétaires se traduisant en ressources et financements limités. Ces contraintes imposent aux professionnelles une redéfinition des priorités et des objectifs dans une logique d'efficacité qui promulgue un maximum de résultats pour un minimum de ressources. 


\section{Des pistes pour l'intervention}

Plusieurs pistes ont été explorées par les Physicians for Human Rights (2000) comme le travail de groupe, l'entraide dans un cadre d'activités et de psychodrame. L'art thérapie a également démontré son efficacité en tant qu'instrument non verbal dans plusieurs groupes à Montréal. (Heusch \& Shermarke 2001). Ailleurs qu'au Canada, des travailleuses sociales ont développé des programmes d'aide aux survivantes. Au Liberia, les participantes d'ateliers sur les conséquences de la violence physique et sexuelle visant la compréhension mutuelle, le soutien et la volonté de défendre les droits des femmes, finirent par s'organiser afin de résoudre elles-mêmes les problèmes de violence sexuelle de leurs camps (Farwell 2004 : 400). Ces types d'interventions nous fournissent un nouveau savoir ainsi que des stratégies innovatrices pour l'intervention et pour la recherche. Ils nous démontrent également l'importance de la congruence des significations et normes culturelles dans la collecte des informations. Du Kosovo où elle a aussi œuvré, Farwell (2004) a rapporté les principes thérapeutiques utilisés par les organisations féministes travaillant avec les survivantes du viol de guerre :

" Les femmes violées doivent être accompagnées par des femmes;

- Les femmes doivent être respectées et leurs histoires crues;

- Les traitements médicaux doivent toujours être accompagnés par des soins sociaux; - L'auto guérison est possible avec l'aide de thérapie appropriée et spécialisée..."
- Les femmes violées doivent être accompagnées par des femmes;

- Les femmes doivent être respectées et leurs histoires crues;

- Les traitements médicaux doivent toujours être accompagnés par des soins sociaux;

- L'auto guérison est possible avec l'aide de thérapie appropriée et spécialisée (Medica Kosova 1999 dans Farwell 2004 : 399).

Le travail des féministes qui se retrouvent en première ligne nous donne un aperçu du savoir-faire et du savoir être dans un contexte de guerre et avec des populations de réfugiées. Les femmes vivent leur viol de guerre ou celui de leurs compatriotes pas seulement comme un crime contre la personne ou comme un problème nécessitant des interventions médicalisées, mais aussi comme une situation découlant du contexte sociopolitique et militaire. C'est ainsi que les organisations de femmes doivent être étroitement orientées vers les services. Elles doivent s'engager dans 
une gamme de stratégies, à partir d'évaluations culturellement sensibles comprenant des soins médicaux et psychosociaux dans des contextes cliniques, mais également communautaires, tout en tenant compte du bien-être économique et de l'importance du soutien féministe (Farwell 2004 : 401).

Nous ne pouvons pas parler d'intersectionnalité sans mentionner l'inclusion des multiples accords et ententes internationales sur les droits humains et les droits des femmes. Il semble nécessaire de toujours contextualiser les expériences dans un contexte de droits humains internationaux et des politiques sociales complexes qui les définissent. Les politiques à la base du cadre législatif incluent la Loi sur la protection des réfugiés et de l'immigration (IRPA) de 2001, les Mesures mises en place depuis le 11 septembre 2001, la Déclaration sur la frontière intelligente - Smart Border Declaration (2001), la Déclaration conjointe sur la coopération des postes de sécurité et de la migration régionale (2001), l'Accord de 2002 sur la sécurité des pays tiers sur les réfugiés entre le Canada et les États-Unis (Oxman-Martinez \& Hanley 2004), les Accords internationaux et les protocoles auxquels le Canada a adhéré, incluant le Comité sur l'élimination de la discrimination contre les femmes (CEDAW), la Convention contre la torture, la Convention de Genève sur le statut de réfugié et les Lignes directrices sur la persécution liée au genre de la Commission de l'immigration et des réfugiés. Les politiques sur la santé et les services de santé aux niveaux provinciaux et municipaux, incluant le logement, le bien-être, les soins spécialisés et les services sociaux pour les victimes de la violence organisée et les politiques sur l'équité dans le milieu de l'emploi sont aussi à considérer.

\section{Conclusion}

Nous avons démontré que le phénomène de la violence de guerre et des conflits ethniques faisait partie d'une toile compliquée de relations qui définissent la place familiale des femmes, leur statut par rapport à leurs enfants, aux hommes et aux aînés, à 
"Il n'existe pas de consensus sur «le comment faire " dans la recension des écrits. ... nous devrions tenir compte des indices de solutions fournis par les femmes ellesmêmes, en considérant leurs contextes de vie avant et après la guerre.» leurs rôles économiques ainsi qu'à leurs obligations religieuses. Il est impérieux que nous centrions nos futures recherches sur la compréhension des contextes des pays d'origine, des codes culturels, des interventions internationales fructueuses, particulièrement dans les contextes de reconstruction comme celui du Rwanda. C'est ainsi que nous serons en mesure de mieux comprendre comment les femmes survivent aux séquelles de la guerre, l'impact du processus de détermination du statut et ses résultats, ainsi que la prévalence du phénomène dans la population de femmes réfugiées et immigrantes venant au Canada.

Il n'existe pas de consensus sur «le comment faire " dans la recension des écrits. Cela renforce l'idée que nous devons éviter des généralisations sur les facteurs culturels et ethniques. Les écrits et nouvelles approches et interventions indiquent que nous devrions tenir compte des indices de solutions fournis par les femmes elles-mêmes, en considérant leurs contextes de vie avant et après la guerre. Cette approche structurelle de l'intervention, en écho aux succès des interventions féministes, valorise les femmes et donc leur résilience. Étant donné les restructurations actuelles des services de santé et des services sociaux, la prise en charge par les femmes de leurs problèmes d'intégration psychosociaux, économiques et politiques leur permet d'exprimer leur volonté de reconstruction de leur vie, selon leur vision, et ce, en dépit de la perte de leurs réseaux familial et communautaire originels.

Le cadre de référence intersectionnel suggéré par la recension des écrits et par notre expérience nous permet d'avancer que les questions de rôles, de statut, de stratification, d'économie, d'ethnicité et d'immigration ne peuvent être élucidées en l'absence de la participation des femmes victimes de viol de guerre et de conflits ethniques et sans tenir compte des sociétés desquelles elles proviennent. Il a de plus été démontré l'urgence d'une analyse critique de nos systèmes de catégorisation et de traitements occidentaux qui, pour le moment et dans la plupart des cas, définissent la maladie et les symptômes post-traumatiques selon des notions biomédicales et uniquement selon un cadre occidental de références. 


\section{Bibliographie}

AFSHAR, Haleh (2003). "Women and wars: some trajectories towards a feminist peace", Development in practice, $13(2-3): 178-188(11)$.

AMNESTY INTERNATIONAL (July 2004). "Sudan, Darfur. Rape as a weapon of war. Sexual violence and its consequences", AFR 54/076/2004.

AMOWITZ, Lynn L., REIS, Chen, LYONS, Kristina Hare, VANN, Beth, MANSARAY, Binta, AKINSULURE-SMITH, Adyinka M., TAYLOR, Louise et Vincent IACOPINO (2002). "Prevalence of War-Related SexualViolence and Other Human Rights Abuses Among Internally Displaced Persons in Sierra Leone”, JAMA, 2002; 287:513-521.

ARCEL, Libby Tata (1998). "Sexual torture of women as a weapon of war - the case of BosniaHerzegovina”, European Psychiatry, 13, Supplément 4:159s-159s (1).

ATER, Richard (1998). "Mental health issues of resettled refugees".

http://ethnomed.org/ethnomed/clin_topics/mental_health.html (page consultée le 2 août 2004)

ATLANI, Laëtitia et Cécile ROUSSEAU (2000). "The politics of culture in humanitarian Aid to Women refugees who have experienced sexual violence", Transcultural Psychiatry. 37(3): 435449.

BAINES, Erin K. (2003). "Body Politics and the Rwandan crisis", Third world Quarterly, 24 (3): 479-493.

BASSOLÉ,Angèle, HAMBOYAN, Hoory, KERISIT, Michèle, PLANTE Nathalie etYOUNG, Marta (2004). "L'impact du conflit armé sur l'intégration des femmes immigrantes et des réfugiées francophones ", Rapport du Mouvement ontarien des Femmes Immigrantes et francophones. (MOFIF), 155p.

BATINIC, Jelena (2001). "Feminism, Nationalism, and War: the Yugoslav Case Feminist Texts”, Journal of International Women's Studies, 3(1):23p. http://www.bridgew.edu/SoAS/jiws/fall01/index.htm (page consultée le 11 juin 2007)

BIEHLER, Anne (2002). "War crimes against women”, Criminal Law Forum, 13:507- 513.

BRITTAIN,Victoria (2003). “The impact of war on women”, Race \& Class, 44(4):41-51.

BUSS, Doris (1998). "Women at the borders: rape and nationalism in international law", Feminist Legal Studies, VI (2):171-203.

BUSS, Doris (2002). "Prosecution Masse Rape: Prosecutor V. Dragoljub Kunarac, Radomir Kovac and Zoran Vukovic", Feminist Legal Studies, 10:91-99.

CARPENTER, R. Charli (2000). "Surfacing children: limitations of genocidal rape discourse", Human Rights Quarterly, 22: 428-477.

CHESTER, Barbara (1992)."Women and Political Torture:Work with Refugee Survivors in Exile", Women Therapy, 13(3): 209-220.

DUCHESNEAU, Claire (2004). "Lessons learned in working with HIV/AIDS african refugees and illegal immigrants in an out-patient setting", Intervention, 120:47-53.

EISENBRUCH, Maurice, de JONG, Joop.T.V.M. et Willem Van de PUT (April/2004). "Bringing order out of chaos: a culturally competent approach to managing the problems of refugees and victims of organized violence", Journal of Traumatic Stress, 17(2):123-131.

FARWELL, Nancy (Winter/2004). "War rape: new conceptualizations and responses", Affilia, 19(4):389-403. 
GARDAM, Judith G. et Hilary CHARLESWORTH (2000). "Protection of Women in Armed Conflict”, Human Rights Quaterly 22(1): 148-166.

HEUSCH, Nicole et Marian SHERMARKE (2001). "Art thérapie et reconstruction identitaire: dévoilement d'expériences traumatiques dans un groupe de femme réfugiées ", PRISME. 35 : $52-70$.

HYNES, H.Patricia (2004/ Nov-Dec.). "On the battlefield of women's bodies: An overview of the harm of war to women”, Women's studies international forum, 27(5-6):431-445.

HYNES, M. et B. LOPES CARDOZO (oct. 2000). "Observations from the CDC: SexualViolence against Refugee Women”, Journal of Women's Health \& Gender-Based Medicine, 9(8): 819 -823.

JACOBSON, Ruth (1999). "Complication 'complexity': integrating gender into the analysis of the Mozambican conflict”, Third World Quarterly - Journal of Emerging Areas, 20(1):175-187(13).

JENNINGS, Peggy, J. et Shauna SWISS (2000). "Statistical Information on violence against Women during the Liberian civil War", Statistics, Development, and human rights: Proceedings of the International Association of Official Statistics, Montreux, Switzerland. 11p.

KIRK, Robin (1992). “Untold terror: violence against women in Peru's armed conflict”, New York, Human Rights Watch.

LACROIX, Marie (2004). “The road to asylum. Canadian refugee policy and the social construction of the refugee claimant subjectivity: Understanding refugeeness", Journal of Refugee Studies, 17(2): 147-166.

LOISELLE-LÉONARD, Margot (2002). « Mort sociale brutale suivie de lente agonie ... souffrances de femmes immigrantes victimes de trafic et de guerre ", Intervention 115:22-32.

McKAY, Susan (2000). "Gender justice and reconciliation”, Women's Studies International Forum, 23 (5):561-570.

MAHMUD, Nasreen (1996). “Crimes Against Honour:Women in International Refugee Law”Journal of refugee Studies, 9(4) : 367-382. MASSON, Sabine (1999). « Le viol en temps de guerre : crime ou bavure? Avancées et résistances de la condamnation du viol contre les femmes. ", Nouvelles questions féministes, 20(3) : 63-79.

NEILL, Kevin Gerard (2000) "Duty, Honor, Rape: Sexual Assault Against women During War", Journal of international women's Studies, 2(1):11pp.

http://www.bridgew.edu/SoAS/JIWS/nov00/duty.htm (page consultée le 11 juin 2007).

NEWLAND, Kathleen (2004). "Seeking protection: Women in asylum and refugee resettlement processes", United Nations; Division for the advancement of women (DAW) 13p. CM/ MMW/2003/EP.8 14 January 2004.

http://www.un.org/womenwatch/daw/meetings/consult/Sweden03docs.htm (page consultée le 11 juin 2007)

NDUWIMANA, Françoise (1999). "Le viol des guerres la riposte des femmes », Relations 655: 269- 272.

NDUWIMANA, F (Décembre 2004). Le droit de survivre : Femmes, violence sexuelle et VIH/SIDA, Droits et Démocratie, Montréal, Canada.

NIARCHOS, Catherine (1995). "Women, War, and Rape: Challenges facing the international Tribunal for the Former Yugoslavia”, Human Rights Quarterly, 17(4): 649-690.

OKAZAWA-REY, Margot (2002). "Warring on Women: Understanding Complex inequalities of gender, race, class, and nation”, Affilia, 17(3):371-383. 
OXMAN-MARTINEZ, Jacqueline, LACROIX, Marie et Jill HANLEY (August 2005), Victims of Trafficking in Persons: Perspectives from the Canadian Community Sector, Department of Justice Canada, Research and Statistics Division. 47 p.

PHYSICIANS for HUMAN RIGHTS (March 2000). "Preliminary Findings and recommendations on the health consequences of human rights violations during the civil war", March 2000 Delegation to Sierra Leone. http://www.phrusa.org/campaigns/sierra_leone/sierra_tripreport. html (page consultée le 24 juillet, 2004

ROJNIK, B., ANDOLSEK-JERAS, L. et D. OBERSNEL-KVEDER (1995). "Women in difficult circumstances: war victims and refugees", International Journal of gynecology E obstetrics, 48(3): 311-315.

ROUSSEAU, Cécile (2000). «Les réfugiés à nos portes : violence organisée et souffrance sociale ", Criminologie, 33(1):185-201.

SALZMAN,T.A (1998). "Rape Camps as a means of Ethnic Cleansing: Religious, Cultural and Ethical Responses to rape victims in the former Yugoslavia", Human Rights Quarterly, 20(2):348-378.

SCHAFER, L. H (2002). “True Survivors: "East African Refugee Women”, Africa Today, 49(2): 29-48.

SHANKS, Leslie et Michael J. SCHULL (2000). "Rape in war: the humanitarian response", Canadian Medical Association Journal, 163(9):1152-1156.

SKJELSBAEK, I (2001). “Sexual violence and war mapping out a complex relationship”, European Journal of International Relations, 7(2):211-237.

SIDERIS, T (2003). "War, gender and culture: Mozambican women refugees", Social Science and Medicine, 56:713-724.

SWISS, S., JENNINGS, P.J.,ARYEE, G., BROWN, G.H., JAPPAH-SAMUKAI, R.M., KAMARA, M.S., SCHAACK, D.H. et R.S. TURAY-KANNEH (1998). "Violence against women during the Liberian civil conflict”, JAMA, 279(8):625-629, February 25.

TURSHEN, M (2000). “The political Economy of violence against women during armed conflict in Uganda”, Social Research, 67(3): 803- 824.

WEAVER, H.N (2005). "Reexamining What We Think We Know: A Lesson Learned From Tamil Refugees”, Affilia,Vol. 20 No. 2, 238-245.

WONG, J.P., LI, A., CHEN, Y.B., KANAGARATNAM, P., YEE, S., FUNG, K. et A.R. SEN Intersecting sexuality, gender, race and citizenship: Mental health issues faced by immigrants and refugees living with HIV/AIDS in Toronto, XVI International AIDS conference, Toronto, Canada, 13-18 August 2006.

\section{Notes}

1. Cet article est basé sur une présentation faite lors du Colloque international, Violences faites aux femmes : réponses sociales plurielles. CRI-VIFF, Montréal, 22, 23, 24 octobre 2006.

2. SelonValtonen (2004) les réfugiés étaient déjà établis dans leur pays d'origine; nous utilisons donc le terme ré établissement pour désigner le processus d'établissement dans le pays d'accueil. 